\title{
Global and distributed torque calculations using the CDSA approach
}

\author{
M. LI, D.A. LOWTHER \\ Department of Electrical and Computer Engineering, McGill University \\ 3480 University Street, Montreal, Quebec, H3A 2A7, Canada \\ e-mail:\{min.li/david.lowther\}@,mcgill.ca
}

(Received: 10.06.2011, revised: 17.09.2011)

\begin{abstract}
Accurate force and torque calculations are fundamental to being able to predict the operation of an electromechanical device or system. The Maxwell stress tensor and the virtual work principle are the two major theories for force and torque calculation. However, if local distributions of torque are needed to couple to structural and vibration analyses, the conventional Maxwell stress approach cannot provide this easily. A recently developed approach based on sensitivity analysis has the capability to deliver local stress and torque as well as accurate global results. In addition, this approach divides the total torque into different components which are essential to the design of electrical devices. This paper includes several numerical examples of torque calculations of different electrical machines. The results are verified by a commercial software package using the Maxwell stress based force calculation.
\end{abstract}

Key words: torque calculation, distributed torques, sensitivity analysis, electrical machines

\section{Introduction}

The accurate computation of electromagnetic forces and torques at both the global and local level is crucial in the design and analysis of electrical machines. In such devices, forces and torques are the mechanism by which electromagnetic energy is translated into mechanical effects (and vice-versa). It is generally accepted that two major approaches exist for the numerical evaluation. The first one is based on the Maxwell stress tensor (MST) which is in the form of a surface integral evaluated in the air region surrounding the body of interest. Numerical examples of MST based torque calculations of electric motors can be found in [1-3]. Although MST force calculation is fairly easy to implement in conjunction with finite element field analysis, the formula relies on the tangential field in the air gap and the resultant torques

\footnotetext{
* This is extended version of a paper which was presented at the 8th International Conference on Computation in Electromagnetics, Wrocław, 11-14.04.2011.
} 
are sensitive to the discretization [3]. Therefore, the integration contour must be carefully chosen in order to avoid the numerical errors in the field solution.

The second category of torque calculation methods is based on the principle of virtual work, where the force or torque on a component in a certain direction can be found by computing the changes in the system energy with respect to a virtual displacement of the body in the same direction. The basic virtual work algorithms require the energy in a system in each of two positions. Coulomb $[4,5]$ first applied the design sensitivity analysis to finite element field analysis and his approach was able to compute the virtual work forces using only one field solution. Later a method based on the direct differentiation of the finite element coenergy was developed by Aronson and Brauer [6]. Local forces can be computed through Kameari's formulation of the virtual nodal displacement with edge elements [7]. Unfortunately, the implementation of the nodal force formulae is quite cumbersome and involves the Jacobian matrix of coordinate transformation which results in a relatively heavy computational cost. Recently a paper by Kim et al. [8] suggested using a continuum design sensitivity analysis (CDSA) approach to determine the virtual work forces through a single solution. The formulation, while based on the values of the fields in the problem, is similar to but differs from the Maxwell stress result in two ways. First, the sensitivity analysis computes a force which is generated whenever a boundary between two materials having different magnetic properties is moved; and, second, since the approach effectively provides a virtual movement of every point individually, it can provide the local stress distribution. In fact, the approach could be applied inside a body wherever there is a change in permeability such as might happen as saturation occurs. This effect cannot be recognized by the Maxwell stress approach as has been pointed out in [9].

The CDSA approach was demonstrated and validated on a simple electrostatic problem in [10], where it has the advantage over MST of not requiring a virtual air gap. The accuracy and mesh dependency issues were discussed in [11].

The goal of the work in this paper is to examine the application of the CDSA virtual work analysis to the problem of a rotating machine. In this case, the torque is needed and the virtual movement of each point in the rotor is in the circumferential direction, i.e. around the center of the rotor unlike the previous work $[8,10,11]$ where all the movement was along one of the main axes. While the CDSA defines a local force component normal to the interface between two different materials, the rotational aspect may lead to some complexity in the implementation. In addition, the sensitivity approach has to be applied along a surface between materials having different permeabilities. For a tooth-slot structure, this means that the evaluation has to pass through areas which have very large fields and, possibly, high levels of numerical error. The Maxwell Stress approach can avoid this because it has to be evaluated along a contour (or surface) in the air region and the contour is generally taken away from the material surface to avoid local errors. The paper first compares the Maxwell stress and CDSA approaches for a simple rotating actuator made of linear material. The second example involves an interior permanent magnet (IPM) motor. The CDSA is able to determine not only the global torques but also the distributed torques in three different areas: torque distributions on the surface of the rotor, on different parts inside the rotor (such as the permanent magnet and the flux bar- 
riers) and the distributions inside the rotor body of saturated nonlinear steel. The capability to predict the distributed torques is particularly important for the design of IPM motors for which we can separate the effects caused by the external excitation or the reluctance change. This can be used for the topological design of the electric motors where the optimal material distribution is to be determined for a desired model of motor.

\section{Torque calculations of electric motors}

The finite element method (FEM) has been widely used to study the behavior of different types of electric motors. With the advances in computing power, the FEM is suitable for solving the electromagnetic field problem of motors with complex structures and efficient force calculation methods have been developed to derive global results from the computed fields.

\section{The MST torque calculation}

The tangential component of the Maxwell stress tensor is used to find the torque density on a rotating part of the motor:

$$
f_{t}=\frac{B_{n} B_{t}}{\mu_{0}}
$$

where $B_{n}$ and $B_{t}$ are the normal and tangential components of the flux density respectively and $\mu_{0}$ is the permeability of air.

The torque is obtained as a surface integral on a contour $l$ surrounding the body in the air:

$$
T=w \cdot\left(r \times \int_{l} f_{t} d l\right),
$$

where $r$ is the radius of the integration contour and $l$ is the length of integration path and $w$ is the width of the 2-D model of the electrical machine.

\section{The virtual work torque calculation}

Torques can also be computed using the virtual work principle as the derivative of the system co-energy $W^{\prime}$ with respect to the displacement angle $\theta$ (under the assumption of constant flux):

$$
T=-\frac{\partial W^{\prime}}{\partial \theta}
$$

The conventional virtual work torque calculation employs a finite difference approach, therefore two FEM field solutions corresponding to the two positions of the rotor are needed.

\section{The CDSA torque calculation}

The CDSA based force calculation method is derived from the shape sensitivity analysis [12] for electromagnetic design problems, which computes the derivative of an objective function $O$ with respect to the boundary shape change. 
Figure 1 shows a two-dimensional magnetostatic problem containing two domains $\Omega_{1}$ and $\Omega_{2}$ with different material properties (e.g. air and iron). $\gamma$ is the interface boundary between $\Omega_{1}$ and $\Omega_{2}$.

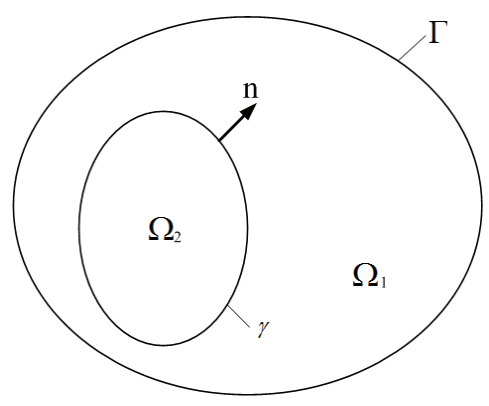

Fig. 1. A 2-D shape design problem

Let us define a scalar function $G$ as an integral over $\Omega_{2}$,

$$
G=\int_{\Omega_{2}} g(x) d \Omega,
$$

where $g(x)$ is a differentiable function of fields defined on $\Omega_{2}$.

We consider both $\Omega_{1}$ and $\Omega_{2}$ as continuum materials, the material derivative [13] of the function $G$ is given as:

$$
G^{\prime}=\int_{\gamma} g(x) V_{n} d \gamma+\int_{\Omega_{2}} g^{\prime}(x) d \Omega
$$

where $V_{n}$ is the normal component of the design velocity which represents the perturbation of the boundary $\gamma$.

The sensitivity of the function $G$ with respect to the variable $p$ can be derived using the adjoint variable method,

$$
\frac{d G}{d p}=\int_{\gamma} L(A, \lambda) V_{n} d \gamma+\int_{\Omega_{2}} \frac{d g}{d p} d \Omega,
$$

where $L(A, \lambda)$ is a function of the magnetic vector potential $A$ and an adjoint variable $\lambda$ determined by $g(x), p$ is the variable describing the boundary change.

Let the system energy, $W$, be the objective function, the problem is self adjoint. We have $A=\lambda$, and there is no need to solve the adjoint problem. Therefore, the sensitivity of the energy with respect to boundary perturbation variables $p$, for a magnetostatic problem, is given in [8] as:

$$
\begin{aligned}
\frac{d W}{d p} & =\int_{\gamma}\left[\left(v_{1}-v_{2}\right) B_{1} \cdot B_{2}\right] V_{n} d \gamma+\int_{\Omega_{2}}\left[\nabla v_{2}\left(B_{2} \cdot B_{2}\right)\right] d \Omega+ \\
& \left.\left.+\int_{\gamma}\left[M_{2}-M_{1}\right] \cdot B_{2}\right] V_{n} d \gamma+\int_{\gamma}\left[J_{2}-J_{1}\right] \cdot A_{2}\right] V_{n} d \gamma,
\end{aligned}
$$


where all values with subscript indices 1 and 2 correspond to the two sub-domains $\Omega_{1}$ and $\Omega_{2}$ containing different materials, $v$ is the material reluctivity, $B$ is the vector of flux density, $A$ is the magnetic vector potential, $M$ is the permanent magnetization, $J$ is the current density.

Therefore, force and torque formulae based on the virtual work principle can be derived using the energy sensitivity in (7). For a ferromagnetic material, the magnetic force consists of a surface force distribution due to the reluctivity change across the surface and a volume force due to the gradient of the reluctivity inside the body $\Omega_{2}$,

$$
T=r \times F=\int_{\gamma} r \times\left[\frac{1}{2}\left(v_{1}-v_{2}\right) B_{1} \cdot B_{2}\right] V_{n} d_{\gamma}+\int_{\Omega_{2}} r \times\left[\frac{1}{2} \nabla v_{2}\left(B_{1} \cdot B_{2}\right)\right] d \Omega .
$$

In addition, the force and torque acting on a permanent magnet is calculated as:

$$
T=r \times F=\int_{\gamma} r \times\left[\left(M_{2}-M_{1}\right) \cdot B_{2}\right] V_{n} d_{\gamma} .
$$

And the force and torque acting on a conductor containing a current is given as:

$$
T=r \times F=\int_{\gamma} r \times\left[\left(J_{2}-J_{1}\right) \cdot A_{2}\right] V_{n} d_{\gamma} .
$$

\section{CDSA force calculation with a finite element mesh}

The CDSA force formulae are often evaluated based on the field solutions from finite element solvers. Figure 2 shows two adjacent triangular elements in a finite element mesh, which are located on the different sides of the interface boundary.

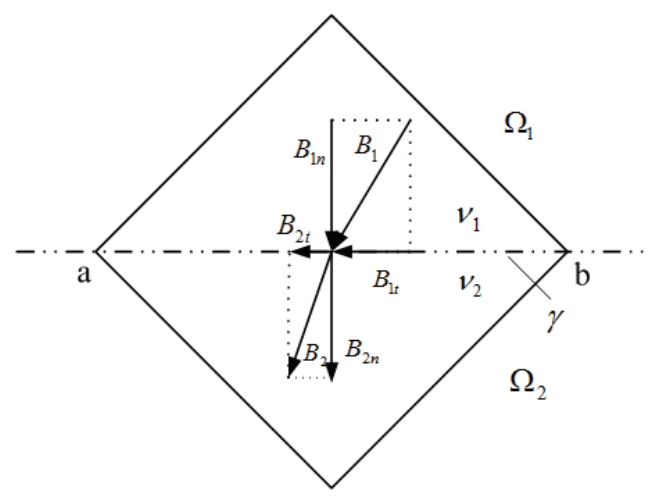

Fig. 2. Fields in adjacent elements

In the following description, all the fields and material properties of one side of the boundary are assigned the subscript 1 and those on the other side of the boundary are assigned the subscript 2.

In the CDSA based force calculation, the unit force exerted on a point on the boundary is given by: 


$$
f_{s}=\frac{1}{2}\left(v_{1}-v_{2}\right) B_{1} \cdot B_{2} .
$$

The flux density $B$ is computed from the magnetic vector potential $A$ from:

$$
B=\nabla \times A .
$$

In each element, $A(x, y)$ is defined as a polynomial function of the coordinates $x$ and $y$. Hence, any local electromagnetic quantity can be evaluated at a given point. The $x$ and $y$ components of the $B$ field are:

$$
\begin{aligned}
& B_{t}=B_{x}=\frac{\partial A(x, y)}{\partial y}, \\
& B_{n}=B_{y}=\frac{\partial A(x, y)}{\partial x} .
\end{aligned}
$$

It is worth noting that the magnetic flux density $B$ changes its angle (direction) while crossing the interface boundary from one material to another. The tangential component of the $B$ field presents a discontinuity on each side of the boundary. Therefore $B_{1}$ and $B_{2}$ have to be obtained from triangular elements on sides 1 and 2 of the boundary respectively.

The total force acting on one element edge is:

$$
\vec{f}_{i}=\frac{1}{2} \int_{a}^{b}\left(v_{1}-v_{2}\right)\left(B_{1 x} B_{2 x}+B_{1 y} B_{2 y}\right) d x .
$$

The global force is obtained by summing up all the local forces:

$$
\vec{F}=\sum_{i=1}^{N} \vec{f}_{i},
$$

where $N$ is the total number of element edges that form the entire boundary of the body.

\section{Comparison between the MST and the CDSA}

The MST and the CDSA both consist of an integral expressed in terms of the magnetic field values, the normal component of the MST is very similar to the surface integral of the CDSA in (8). However, the CDSA integration is taken on the interface between the iron and air while the integration contour of the MST must be taken in the air region. In addition, the CDSA does not require a closed contour in the air, thus it can be applied to a boundary between any two materials having different magnetic properties.

\section{Numerical examples}

Two numerical models for torque calculation were studied. Both models were solved using the commercial finite element field analysis software, MagNet [14]. The MST torques were 
provided by MagNet using a tunable volume integration formulation based on MST [15]. The CDSA torques were computed using exactly the same field solutions.

\section{Example 1 - Torques on a rotating block}

The first model for the CDSA torque verification consists of a simple C-core and a rotating block (Fig. 3). The core and the rotating block are both made of a linear material with a relative permeability of 1000 .

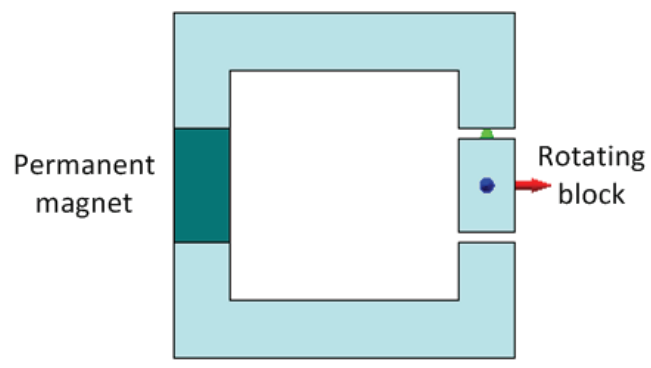

Fig. 3. C-core with a rotating block

The surface force distributions produced by the CDSA approach are illustrated in Fig. 4 for two different positions of the block. According to the CDSA, large forces are distributed at the corner of the block where high magnetic fields are present.

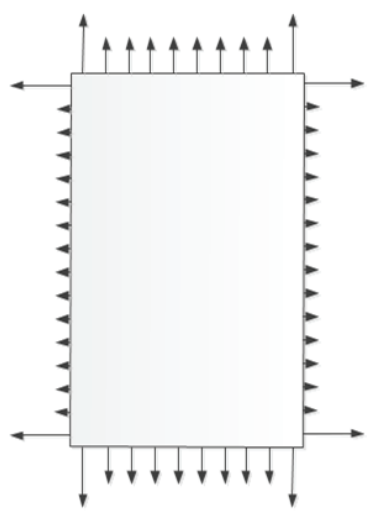

a) Rotation angle $=0$ degree

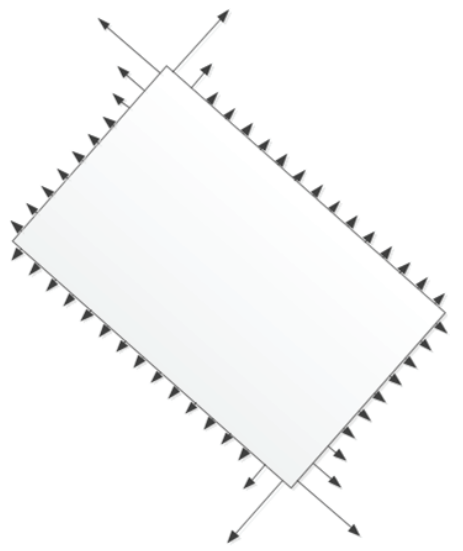

b) Rotation angle $=45$ degree

Fig. 4. Force distributions by the CDSA

The total torque of the CDSA is obtained by integrating the distributed torque on the boundary of the rotating block. The CDSA torques are computed at seven positions of the block: $0,15,30,45,60,75,90$ degrees and are compared to the results provided by MagNet in 
Fig. 5. We can observe a good agreement between the two results, and the slight discrepancy may be a result of the numerical errors in the field solutions.

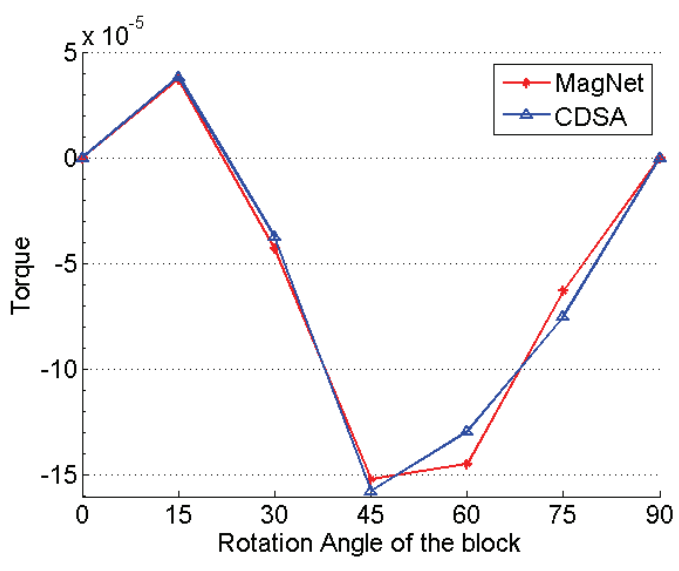

Fig. 5. Torques vs. rotation angles

\section{Example 2 - Torques of an IPM machine}

This example looks at the torque calculation generated by a 3-phase, 4-pole interior permanent magnet (IPM) machine. The detailed structure of the IPM machine is shown in Figure 6. Only a quarter of the motor is modeled in order to reduce the computational cost. The rotor contains three different materials, a $\mathrm{NdFeB}$ (neodymium iron boron) magnet bar, two flux barriers of air, and the rest of the rotor is made of M19 steel, which is also used in the stator.

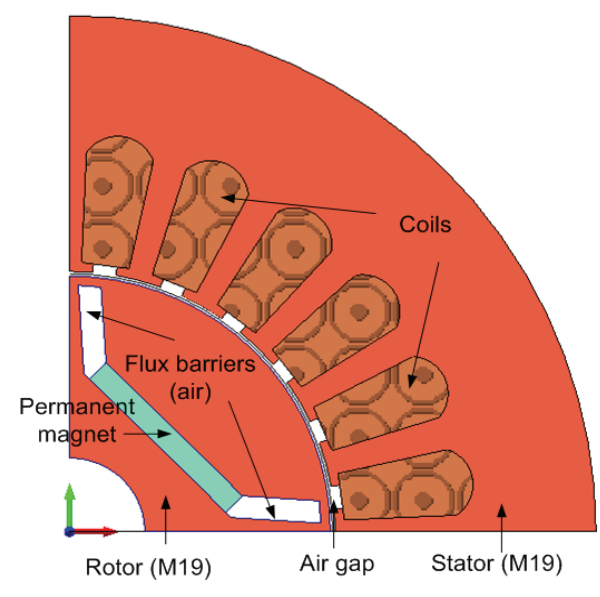

Fig. 6. A quarter of an IPM machine

Figure 7 shows the geometries of the IPM components. The values of all the parameters are given in Table 1. 


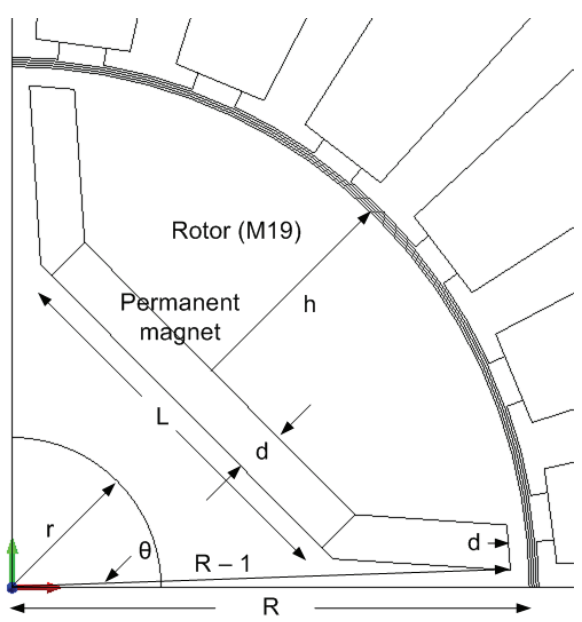

Fig. 7. Geometries of the IPM motor

Table 1. Parameters of the IPM motor

\begin{tabular}{c|c}
\hline Symbol & Value \\
\hline$R$ & $27.75 \mathrm{~mm}$ \\
\hline$r$ & $8 \mathrm{~mm}$ \\
\hline$L$ & $20.5 \mathrm{~mm}$ \\
\hline$d$ & $2.5 \mathrm{~mm}$ \\
\hline$h$ & $12 \mathrm{~mm}$ \\
\hline$\theta$ & $2 \mathrm{deg}$ \\
\hline
\end{tabular}

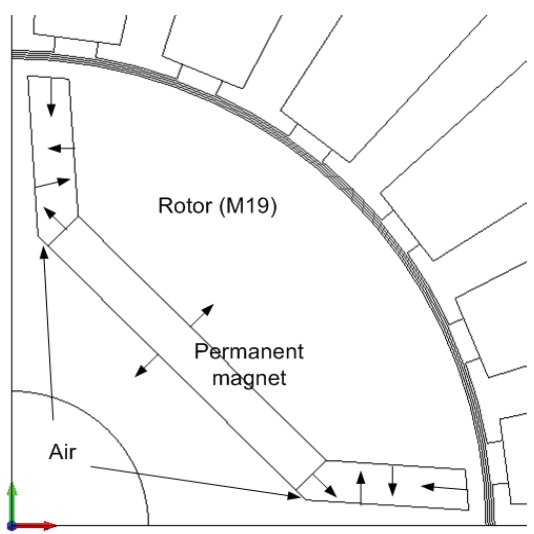

Fig. 8. Illustration of local force directions

Unlike the MST torque calculation, the CDSA is able to determine the local force distributions between any boundaries of two different materials (e.g. iron and air or permanent magnet and iron). The directions of the distributed forces inside the rotor are indicated in 
Figure 8. The global torque is obtained as the summation of all local distributed torques on the rotor surface and inside the rotor body.

The IPM motor model is solved for 31 positions of the rotor, starting at zero degrees and increasing at one degree each time. Torques are computed at each position and plotted against the rotor angle.

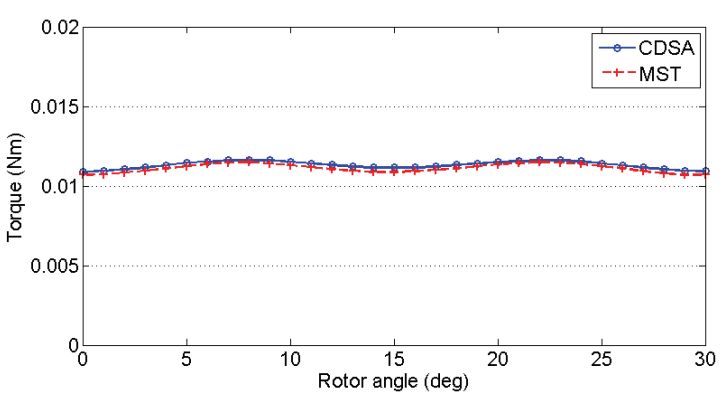

Fig. 9. Torques against rotor position of a linear core

If a linear material is used instead of the M19 in the rotor, the volume integral in the CDSA disappears, thus only the surface torques distributed between the different components of the rotor account for the global torque. As shown in Figure 9, the CDSA torques agrees with the MST torque in every position.

In reality, part of the rotor of nonlinear material is saturated as shown in Figure 10. Forces distributed between the saturated and non-saturated areas cannot be ignored.

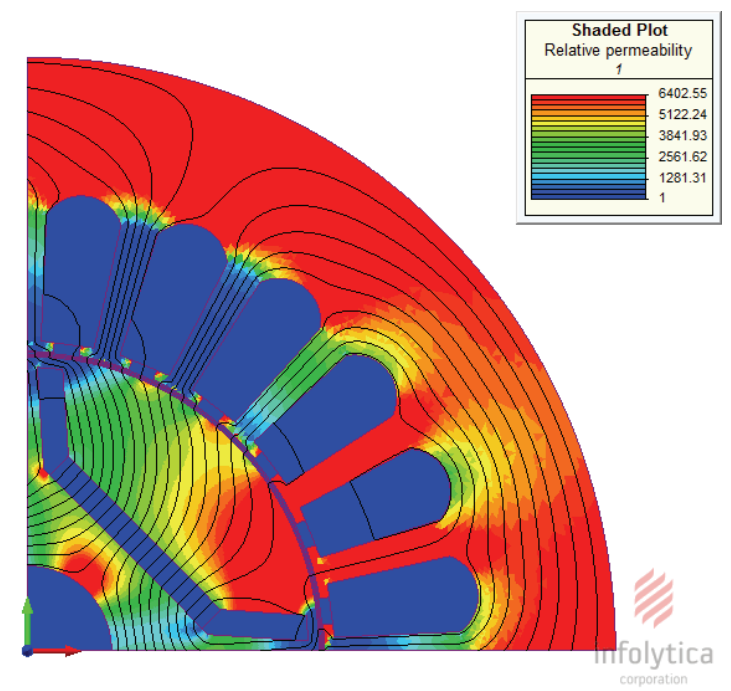

Fig. 10. Plot of relative permeability distribution 
Figure 11 shows the comparison of the MST torques and the CDSA torques computed from the surface integral for a non-linear rotor core. Large discrepancies are observed and this results from the reluctance torque due to the saturation in the material.

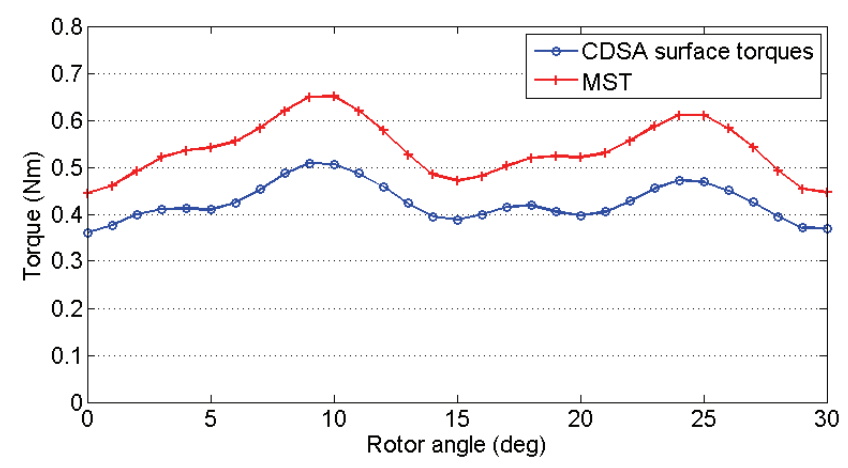

Fig. 11. Torques against rotor position of a non-linear core

The CDSA volume torques are evaluated using a fine mesh. The model has a total of more than 90000 elements in the rotor where the maximum element size is set to $0.1 \mathrm{~mm}$. In order to simplify the calculation, we used first order elements inside which the relative permeability is a constant. The distributed torques are computed on the three sides of every element in the mesh. The volume torques are shown in Figure 12 for 31 positions of the rotor.

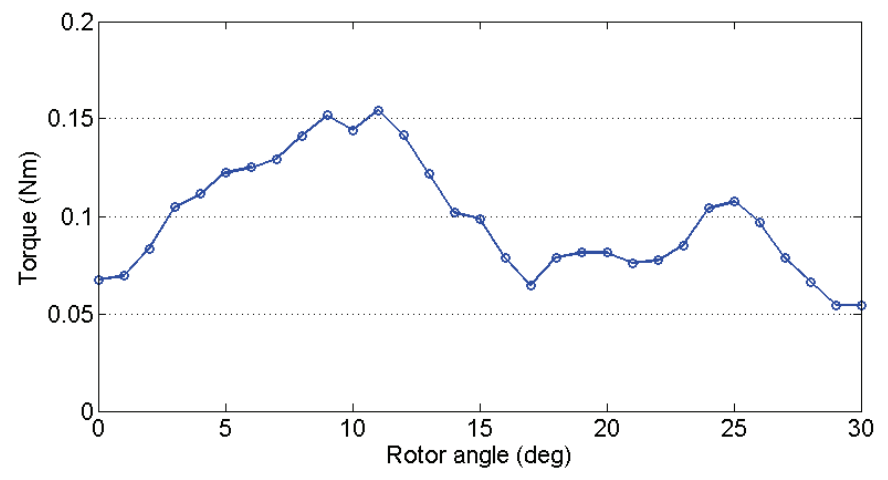

Fig. 12. Volume torques against rotor position

The combination of the surface torque and the volume torque makes up the CDSA total torque. The CDSA total torques are compared with the MST torques in Figure 13. As we can see, the volume torque has made up for the discrepancy and the results of the two approaches are very close. The small difference between the two torques for rotor angles after 16 degrees may be result from the discretization error of the finite element model. 


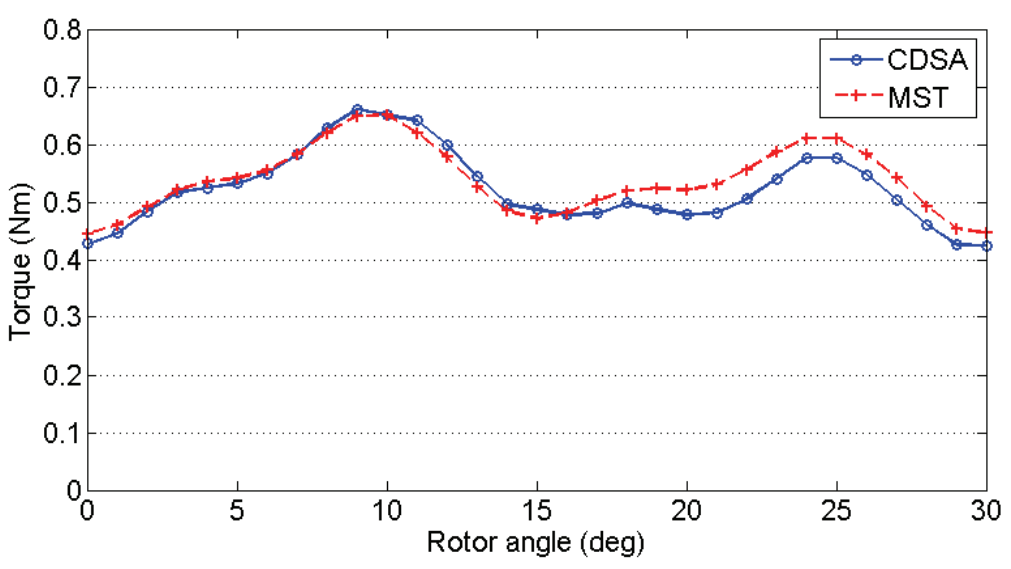

Fig. 13. MST vs. CDSA total torques

\section{Conclusion}

A CDSA based method is applied to the calculation of torques of electrical machines. The results are verified by MagNet which uses a MST based torque calculation. Unlike the MST, the CDSA force calculation method can be applied directly to the boundary. The CDSA method also produces the surface force distributions and internal force distributions inside a body where different materials exist. Thus this method has the potential to be applied to coupled structural-magnetic analysis problems such as are encountered in deformation and vibration computations.

In the case of a non-linear problem, the CDSA needs to evaluate both the surface integral and the volume integral for the total torque while the MST torque calculation is relatively simpler. On the other hand, the CDSA torque formulation defines different components of the torque. The volume integral accounts for the torque caused by the reluctance change inside the rotor due to the saturation. It provides an important piece of information for the design optimization of IPM and other motors.

Finally, the evaluation of the CDSA torque is as efficient as that of the MST. And we may use the subtraction of the CDSA surface integral from the MST torque to compute the torque due to internal permeability changes indirectly and thus avoiding the direct evaluation of the volume integral. However, if this is done, the internal distribution of local torques cannot be determined.

\section{Acknowledgements}

This work was supported by the Natural Science and Engineering Research Council of Canada and Le Fonds Quebecois de la Recherche sur la Nature et les Technologies. 


\section{References}

[1] Mizia J., Adamiak K. et al., Finite element force calculation: comparison of methods for electric machines. Magnetics, IEEE Transactions on Magnetics 24(1): 447-450 (1988).

[2] Howe D., Zhu Z.Q., The influence of finite element discretisation on the prediction of cogging torque in permanent magnet excited motors. IEEE Transactions on Magnetics 28(2): 1080-1083 (1992).

[3] Salon S., Bhatia S. et al., Some aspects of torque calculations in electrical machines. IEEE Transactions on Magnetics 33(2): 2018-2021 (1997).

[4] Coulomb J., A methodology for the determination of global electromechanical quantities from a finite element analysis and its application to the evaluation of magnetic forces, torques and stiffness. IEEE Transactions on Magnetics 19(6): 2514-2519 (1983).

[5] Coulomb J., Meunier G., Finite element implementation of virtual work principle for magnetic or electric force and torque computation. IEEE Transactions on Magnetics 20(5): 1894-1896 (1984).

[6] Aronson E.A., Brauer J.R., Magnetic torque or force calculation by direct differentiation of finite element coenergy. IEEE Transactions on Magnetics 25(5): 3578-3580 (1989).

[7] Kameari A., Local force calculation in 3D FEM with edge elements, in Nonlinear Phenomena in Electromagnetic Fields. Amsterdam, The Netherlands, Elsevier 449-452 (1992).

[8] Kim D.-H. et al., Efficient global and local force calculations based on continuum sensitivity analysis. IEEE Transactions on Magnetics 43: 1177-1180 (2007).

[9] Bossavit A., Virtual Power Principle and Maxwell's tensor: Which comes first? Proceedings of the14th IGTE Symposium, Graz, Austria, September 2010, pp 46 (2010).

[10] Li M., Kim D.-H., Lowther D.A., Sykulski J.K., A sensitivity approach to force calculation in electrostatic MEMS Devices. IEEE Transactions on Magnetics 44: 1610-1613 (2008).

[11] Li M., Lowther D.A., Local electromagnetic force computation in the presence of numerical field errors. IEEE Transactions on Magnetics 45(3) 1344-1347 (2009).

[12] Park I.H., Coulomb J.L. et al., Implementation of continuum sensitivity analysis with existing finite element code. IEEE Transactions on Magnetics 29(2), 1787-1790 (1993).

[13] Haug E.J., Choi K.K., Komkov V., Design sensitivity analysis of structural systems. Academic Press, New York, N.Y. (1986).

[14] MagNet user's manual. http://www.infolytica.ca (2011).

[15] McFee S., Webb J.P. et al., A tunable volume integration formulation for force calculation in finite-element based computational magnetostatics. IEEE Transactions on Magnetics 24(1): 439-442 (1988). 